\title{
Organochlorine Pesticide Residues Level in Fish, Water and Sediments in South-South Nigeria
}

\author{
Omoruyi $\mathrm{K}^{1 *}$, Abolagba $\mathrm{OJ}^{1}$, Igene $\mathrm{JO}^{2}$, Usifoh $\mathrm{SF}^{3}$ and Usifoh $\mathrm{CO}^{4}$ \\ ${ }^{1}$ Department of Aquaculture and Fisheries Management, Faculty of Agriculture, University of Benin, Benin City, Nigeria \\ ${ }^{2}$ Department of Food Science Technology, Faculty of Agriculture, University of Benin, Benin City, Nigeria \\ ${ }^{3}$ Department of Clinical Pharmacy, Faculty of Pharmacy, University of Benin, Benin City, Nigeria \\ ${ }^{4}$ Department of Pharmaceutical Chemistry, Faculty of Pharmacy, University of Benin, Benin City, Nigeria
}

\section{*Corresponding Author}

Omoruyi K

\section{Article History}

Received: 12.10 .2020

Accepted: 28.10 .2020

Published: 17.11.2020

\begin{abstract}
This study reveals the distribution and levels of the organochlorine pesticide residues in fish, water and sediments in South-South, Nigeria. They were extracted with dichloromethane and screened for organochlorine pesticides with Gas Chromatography-Mass Spectrophotometry (GC-MS). All data collected were exposed to analysis of variance at $(\mathrm{p}<0.05)$ and the means were separated by means of Duncan Multiple Range Test (DMRT). It was discovered in this study that organochlorine pesticide residues were not discovered in all the fish and water sampled, while there was significant difference $(\mathrm{P}<0.05)$ in the levels of some organochlorine pesticides detected in the sediments. Sediment samples from Oron in Akwa-Ibom State recorded $1.50 \pm 0.25 \mu \mathrm{g} / \mathrm{kg}$ and $30.80 \pm 0.20 \mu \mathrm{g} / \mathrm{kg}$ for lindane and aldrin residues respectively; Mkpana Beach (Akwa-Ibom State) had lindane $(3.70 \pm 0.20 \mu \mathrm{g} / \mathrm{kg}$ ), aldrin $(4.20 \pm 0.20 \mu \mathrm{g} / \mathrm{kg})$, endosulfan $(0.60 \pm 0.30 \mu \mathrm{g} / \mathrm{kg})$ and ortho-para-dichloro-diphenyl-trichloro ethane (o,p'- DDT) $(0.20 \pm 0.10 \mu \mathrm{g} / \mathrm{kg})$. Illushin and Agenebode in Edo State had $0.10 \pm 0.02 \mu \mathrm{g} / \mathrm{kg}$ and $1.00 \pm 0.30 \mu \mathrm{g} / \mathrm{kg}$ of aldrin residues correspondingly. They were all above the allowable limit of $0.05 \mu \mathrm{g} / \mathrm{kg}$ recommended by Environmental Protection Agency (EPA). The pesticide-free fish and water could be as a result of the fact that the Fisher folks no longer use pesticides in fishing due to the awareness of the imminent dangers involved. However, the high levels of Pesticide residues in sediments may be as a result of overtime bioaccumulation of extensive and repeated use of the pesticides in fishing.
\end{abstract}

Keywords: Organochlorine Pesticide; Fish, Sediment; Water; GC/MS; South-South Nigeria.

\section{INTRODUCTION}

Pesticide residues are substances in foods meant for man or animals consequential from the use of pesticide comprising of derivatives, such as impurities and contaminants known to be hazardous [1]. Organochlorines are tenacious contaminants that have triggered global attention as one of the most toxic environmental pollutants [2]. They are lipophillic, have affinity for water and are found everywhere because of their persistence in nature, stemming from water currents and other anthropogenic passageways [3]. Organochlorine pesticides have extensively used in the world and have been found in foodstuffs, water, fish and sediments $[4,5]$.

The production as well as usage of most pesticides have been proscribed in most nations but are still in use by majority of the uncivilized nations by health and agricultural sectors [6].

Most fishermen, farmers have abused their use for the purpose of agriculture [4]. Chlorinated pesticides residues can enter aquatic ecosystem via discharges of effluents and domestic sewage and industrial wastewater and so on [7]. Pesticide residues could be dispersed amongst water sediments and fish components of the ecosystem. Most studies on humans and experimental animals prove the toxic potential of contact to organochlorine pesticides $[8,9]$.

This study was therefore aimed at determining the amounts and distributions of organochlorines residues in fish, water and sediments in South-South, Nigeria.

Copyright $\odot 2020$ The Author(s): This is an open-access article distributed under the terms of the Creative Commons Attribution 4.0 International License (CC BY-NC 4.0) which permits unrestricted use, distribution, and reproduction in any medium for noncommercial use provided the original author and source are credited. 


\section{Materials ANd Methods}

\section{Collection of fish Samples}

Fish samples were captured with the assistance of artisanal fishermen using local fish traps, cast nets and baited hooks. Fish were washed in flowing water to eliminate adhering debris before they were conveyed to the laboratory within 24 hours in an ice chest.

\section{Collection of Water samples}

Surface water samples were taken from three sampling stations (1-downstream, 2-midstream and 3-upstream) from each water body at $30 \mathrm{~cm}$ depth into amber glass bottles $(2 \mathrm{ml})$ already washed using detergents, rinsed with distilled water, soaked overnight in $10 \%$ nitric acid and finally rinsed using distilled water. Water samples were taking from three sampling stations each from Koko, Ekehuan, Illushin, Agenebode, Old Nigeria Port Authority (NPA), Brass, Choba, Oron water bodies. All the samples of water were acidified using $5 \mathrm{ml}$ of $10 \%$ nitric acid to minimize adsorption of heavy metals to the walls of the plastic bottles [10].

\section{Collection of Sediment Samples}

Samples of sediment were taken from three sampling stations of the selected water bodies in the study areas using an Eckman's grab to get and put the samples into plastic bags. The plastic bags were initially cleaned using detergents and treated with $10 \%$ nitric acid. They were dehydrated at $105^{\circ} \mathrm{C}$ in a kiln to a constant weight and ground to powder. The powder samples were sieved through a $0.5 \mathrm{~mm}$ sieve to remove ungrounded materials. $1 \mathrm{~g}$ of the fine filtrate was digested and subsequently analyzed [10].

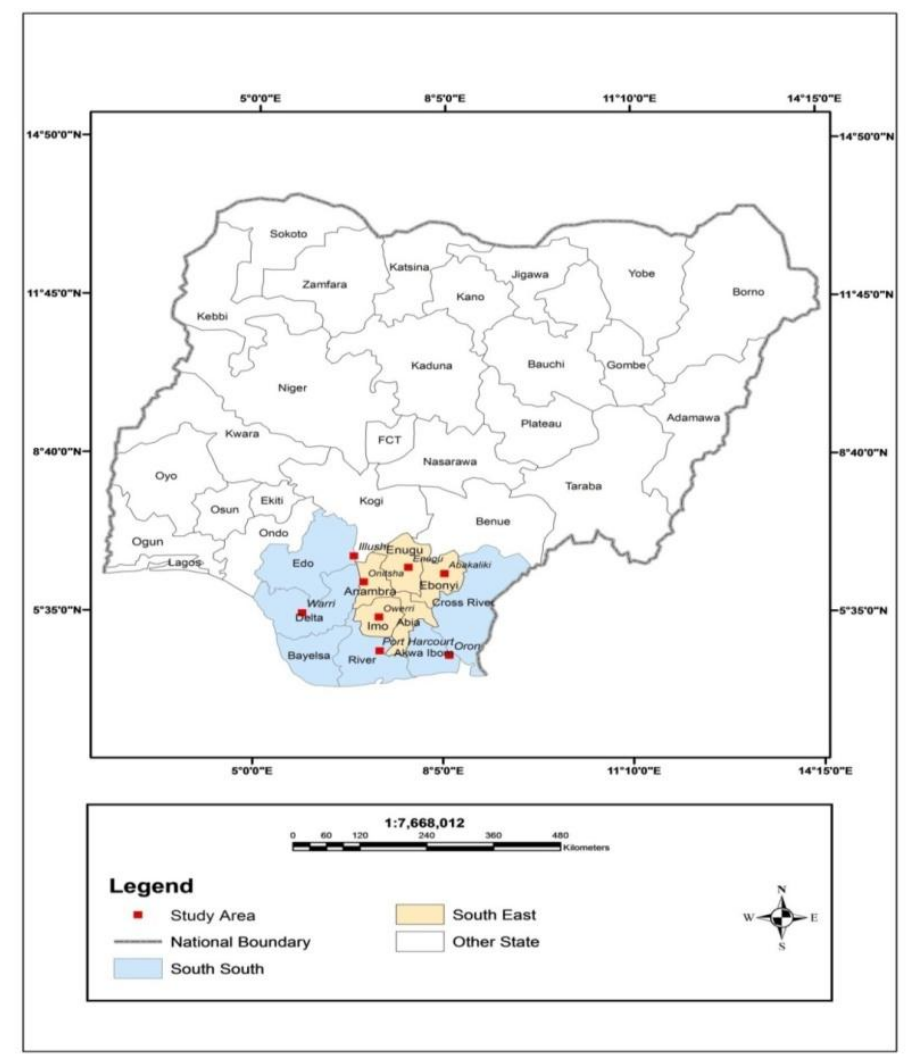

\section{Extraction Procedure of Water Samples}

Water sample of $250 \mathrm{ml}$ was measured into an already washed separating funnel. $20 \mathrm{ml}$ of dichloromethane was poured in the separating funnel, after which, it was covered and vigorously shaken for 30 mins while the cover of the separating funnel was open and closed intermittently to expel gas from it. The extract was then recovered from dichloromethane and the process was repeated thrice to ensure complete extraction [11]. The extract was taken for cleaning to remove impurities.

\section{Extraction Procedure of Sediment Samples}

The grounded sample of $10 \mathrm{~g}$ was extracted in a Soxhlet apparatus with $150 \mathrm{ml}$ dichloromethane for $3 \mathrm{hrs}$. at a temperature of $40^{\circ} \mathrm{C}$ [12]. The dichloromethane containing the extract was left overnight to dry. The extract was weighed and taken for cleaning. 


\section{Extraction Procedure of Fish Samples}

The crushed sample of $20 \mathrm{~g}$ was extracted in a Soxhlet apparatus with $150 \mathrm{ml}$ dichloromethane for $3 \mathrm{hrs}$. at a temperature of $40^{\circ} \mathrm{C}$ [12]. The dichloromethane containing the extract was left overnight to dry. The extract was weighed and taken for cleaning.

\section{Clean-up Procedures of the Extracts}

The extract from fish, water and sediment samples were cleaned-up (Solid Phase Extraction) using a short column $(15 \mathrm{~cm})$ diameter silica gel $(200-400 \mu \mathrm{m})$ particles mesh size with dichloromethane $\left(\mathrm{CH}_{2} \mathrm{Cl}_{2}\right)$ as the mobile phase.

Silica gel of $10 \mathrm{~g}$ prepared in slurry form and $10 \mathrm{~g}$ anhydrous sodium sulphate was inserted in the column to grip water from the extract. The extract was put in the column and sinks through the sodium sulphate and silica gel packed layers. Elution was done with $20 \mathrm{ml}$ dichloromethane. The elute was pipetted into plastic vessels and evapourated to dryness under a stream of analytical grade nitrogen in readiness for GC-MS [12].

\section{GC-MS}

GC-MS was employed to identify and quantify the residues of organochlorine in the extracts replicated from the cured-fish samples. A Shimadzu GC-MS P-2010 was employed to classify specified compounds, built with split/splitless injector and Restek Stx-Cl pesticide column (Length $30 \mathrm{~m}, 0.25 \mathrm{~mm}$ I.D, and $0.25 \mu \mathrm{m}$ thickness). Electron ionization mass spectra were generated at $70 \mathrm{eV}$, monitoring for ions $\mathrm{m} / \mathrm{z} 50$ to 450 in full-scan as well as designated ion record mode. Injection volume and temperature were $1 \mu \mathrm{L}$ and $250{ }^{\circ} \mathrm{C}$ respectively. Interface and iron source temperature are $250{ }^{\circ} \mathrm{C}$ and $200{ }^{\circ} \mathrm{C}$ respectively, injection temperature and detection hotness were set as $250{ }^{\circ} \mathrm{C}$. while that of the column was set as $60{ }^{\circ} \mathrm{C}$ and increased immediately at the rate of $10{ }^{\circ} \mathrm{C} / \mathrm{min}$. to $180{ }^{\circ} \mathrm{C}$ where it was seized for $2 \mathrm{~min}$, this was improved by $15{ }^{0} \mathrm{C} / \mathrm{min}$., to $280{ }^{\circ} \mathrm{C}$, seized finally for $4 \mathrm{~min}$.

\section{STATISTICAL ANALYSIS}

A GENSTAT® computer software (Version 13.1 for Windows) was used for statistical analysis. Analysis of variance (ANOVA) was used to test for significant differences $(P<0.05)$ between mean values of metals while Duncan Multiple Range Test (DMRT) was used to separate significant means.

Tabl-1: Analysis of fresh fish samples from some water bodies in South-South

\begin{tabular}{|c|c|c|c|c|c|c|}
\hline $\mathbf{S} / \mathbf{N}$ & Water bodies & $\begin{array}{c}\text { Sampling } \\
\text { Stations }\end{array}$ & Sample Name & $\begin{array}{c}\text { Ave. wt of } \\
\text { whole Fish (g) }\end{array}$ & $\begin{array}{l}\text { Wt of Ext. Analyzed } \\
\text { with GC/MS (g) }\end{array}$ & $\begin{array}{l}\text { Pesticides/Con } \\
\text { c. Found } \\
\end{array}$ \\
\hline 1 & Ekehuan & $\begin{array}{l}1 \\
2 \\
3\end{array}$ & $\begin{array}{l}\text { C. } \text { gariepinus } \\
\text { P. obscura } \\
\text { S. clarias }\end{array}$ & $\begin{array}{l}365 \\
459 \\
320\end{array}$ & $\begin{array}{l}0.44 \\
0.65 \\
0.52\end{array}$ & $\begin{array}{l}\text { N.D } \\
\text { N.D } \\
\text { N.D }\end{array}$ \\
\hline 2 & Illushin & $\begin{array}{l}1 \\
2 \\
3\end{array}$ & $\begin{array}{l}\text { C. gariepinus } \\
\text { E. fimbriata } \\
P . \text { obscura }\end{array}$ & $\begin{array}{l}600 \\
250 \\
355\end{array}$ & $\begin{array}{l}0.80 \\
0.76 \\
0.52\end{array}$ & $\begin{array}{l}\text { N.D } \\
\text { N.D } \\
\text { N.D }\end{array}$ \\
\hline 3 & Agenebode & $\begin{array}{l}1 \\
2 \\
3\end{array}$ & $\begin{array}{l}P . \text { obscura } \\
\text { S. clarias } \\
\text { C. senegalensis }\end{array}$ & $\begin{array}{l}420 \\
463 \\
250\end{array}$ & $\begin{array}{l}0.56 \\
0.64 \\
0.51\end{array}$ & $\begin{array}{l}\text { N.D } \\
\text { N.D } \\
\text { N.D }\end{array}$ \\
\hline 4 & $\begin{array}{l}\text { Old NPA } \\
\text { (Warri) }\end{array}$ & $\begin{array}{l}1 \\
2 \\
3\end{array}$ & $\begin{array}{l}\text { S. clarias } \\
\text { H. niloticus } \\
\text { P.senegalensis }\end{array}$ & $\begin{array}{l}405 \\
900 \\
400\end{array}$ & $\begin{array}{l}0.98 \\
0.46 \\
0.66\end{array}$ & $\begin{array}{l}\text { N.D } \\
\text { N.D } \\
\text { N.D }\end{array}$ \\
\hline 5 & Koko & $\begin{array}{l}1 \\
2 \\
3\end{array}$ & $\begin{array}{l}\text { C. senegalensis } \\
\text { P.senegalensis } \\
\text { E. fimbriata }\end{array}$ & $\begin{array}{l}310 \\
290 \\
320\end{array}$ & $\begin{array}{l}0.57 \\
0.76 \\
0.86\end{array}$ & $\begin{array}{l}\text { N.D } \\
\text { N.D } \\
\text { N.D }\end{array}$ \\
\hline 6 & Oron & $\begin{array}{l}1 \\
2 \\
3 \\
\end{array}$ & $\begin{array}{l}\text { E. fimbriata } \\
\text { C. senegalensis } \\
\text { P.senegalensis }\end{array}$ & $\begin{array}{l}450 \\
290 \\
195 \\
\end{array}$ & $\begin{array}{l}0.68 \\
0.50 \\
0.70 \\
\end{array}$ & $\begin{array}{l}\text { N.D } \\
\text { N.D } \\
\text { N.D } \\
\end{array}$ \\
\hline 7 & Choba & $\begin{array}{l}1 \\
2 \\
3\end{array}$ & $\begin{array}{l}\text { H. niloticus } \\
\text { C. gariepinus } \\
\text { S. clarias }\end{array}$ & $\begin{array}{l}545 \\
654 \\
343\end{array}$ & $\begin{array}{l}0.85 \\
0.80 \\
0.45 \\
\end{array}$ & $\begin{array}{l}\text { N.D } \\
\text { N.D } \\
\text { N.D }\end{array}$ \\
\hline 8 & $\begin{array}{c}\text { Mkpana } \\
\text { (Ibeno LGA) }\end{array}$ & $\begin{array}{l}1 \\
2 \\
3\end{array}$ & $\begin{array}{l}\text { P.senegalensis } \\
\text { C. senegalensis } \\
\text { E. fimbriata }\end{array}$ & $\begin{array}{l}255 \\
321 \\
254\end{array}$ & $\begin{array}{l}0.76 \\
0.65 \\
0.56\end{array}$ & $\begin{array}{l}\text { N.D } \\
\text { N.D } \\
\text { N.D }\end{array}$ \\
\hline 9 & $\begin{array}{c}\text { Brass } \\
\text { (Yenegoa) }\end{array}$ & $\begin{array}{l}1 \\
2 \\
3\end{array}$ & $\begin{array}{l}\text { C. gariepinus } \\
\text { S. clarias } \\
\text { H. niloticus }\end{array}$ & $\begin{array}{l}250 \\
435 \\
565\end{array}$ & $\begin{array}{l}0.78 \\
0.50 \\
0.60\end{array}$ & $\begin{array}{l}\text { N.D } \\
\text { N.D } \\
\text { N.D }\end{array}$ \\
\hline
\end{tabular}

$$
\text { * N.D - Not Detected }
$$




\section{RESULTS}

The results of the organochlorine residues analysed in the fish, water and sediment samples in South-South Nigeria are shown in Tables I - III.

Analyses of fresh fish samples from some water bodies in South-South

It can be seen in Table I that no organochlorine pesticide was detected in fresh fish samples from South-South Nigeria.

\section{Analysis of Organochlorine Residues in the Water from South-South}

The analysis of organochlorine residues in the water samples from South-South Nigeria are presented in the Tables II. The study revealed that there were no residues of organochlorine in water.

Table-2: Concentrations of Organochlorine Pesticide Residues in some Water Bodies in South-South

\begin{tabular}{|c|c|c|c|c|c|c|c|c|}
\hline SN & $\begin{array}{l}\text { Water } \\
\text { bodies }\end{array}$ & $\begin{array}{l}\text { Sampling } \\
\text { stations }\end{array}$ & $\mathbf{P}^{\mathrm{H}}$ & $\begin{array}{l}\text { Wt of } \\
\text { Extract } \\
\text { (g) }\end{array}$ & $\begin{array}{l}\text { Lindane } \\
\text { Conc. } \\
(\mu \mathrm{g} 1)\end{array}$ & $\begin{array}{l}\text { Aldrin } \\
\text { Conc. } \\
(\mu \mathrm{g} /)\end{array}$ & $\begin{array}{l}\text { Endosulfan } \\
\text { Conc. } \\
(\mu \mathrm{g} 1)\end{array}$ & $\begin{array}{l}\text { DDT } \\
\text { Conc. } \\
(\mu \mathrm{g} 1)\end{array}$ \\
\hline 1 & Ekehuan & $\begin{array}{l}1 \\
2 \\
3\end{array}$ & $\begin{array}{l}6.3 \\
6.5 \\
6.0\end{array}$ & $\begin{array}{l}0.03 \\
0.02 \\
0.03\end{array}$ & $\begin{array}{l}\text { N.D } \\
\text { N.D } \\
\text { N.D }\end{array}$ & $\begin{array}{l}\text { N.D } \\
\text { N.D } \\
\text { N.D }\end{array}$ & $\begin{array}{l}\text { N.D } \\
\text { N.D } \\
\text { N.D }\end{array}$ & $\begin{array}{l}\text { N.D } \\
\text { N.D } \\
\text { N.D }\end{array}$ \\
\hline 2 & Illushin & $\begin{array}{l}1 \\
2 \\
3\end{array}$ & $\begin{array}{l}5.9 \\
6.2 \\
5.8\end{array}$ & $\begin{array}{l}0.02 \\
0.04 \\
0.02\end{array}$ & $\begin{array}{l}\text { N.D } \\
\text { N.D } \\
\text { N.D }\end{array}$ & $\begin{array}{l}\text { N.D } \\
\text { N.D } \\
\text { N.D }\end{array}$ & $\begin{array}{l}\text { N.D } \\
\text { N.D } \\
\text { N.D }\end{array}$ & $\begin{array}{l}\text { N.D } \\
\text { N.D } \\
\text { N.D }\end{array}$ \\
\hline 3 & Agenebode & $\begin{array}{l}1 \\
2 \\
3\end{array}$ & $\begin{array}{l}6.5 \\
6.8 \\
6.1 \\
\end{array}$ & $\begin{array}{l}0.02 \\
0.06 \\
0.05\end{array}$ & $\begin{array}{l}\text { N.D } \\
\text { N.D } \\
\text { N.D }\end{array}$ & $\begin{array}{l}\text { N.D } \\
\text { N.D } \\
\text { N.D }\end{array}$ & $\begin{array}{l}\text { N.D } \\
\text { N.D } \\
\text { N.D }\end{array}$ & $\begin{array}{l}\text { N.D } \\
\text { N.D } \\
\text { N.D }\end{array}$ \\
\hline 4 & $\begin{array}{l}\text { Old NPA } \\
\text { (Warri) }\end{array}$ & $\begin{array}{l}1 \\
2 \\
3\end{array}$ & $\begin{array}{l}7.8 \\
6.5 \\
6.6\end{array}$ & $\begin{array}{l}0.02 \\
0.02 \\
0.03\end{array}$ & $\begin{array}{l}\text { N.D } \\
\text { N.D } \\
\text { N.D }\end{array}$ & $\begin{array}{l}\text { N.D } \\
\text { N.D } \\
\text { N.D }\end{array}$ & $\begin{array}{l}\text { N.D } \\
\text { N.D } \\
\text { N.D }\end{array}$ & $\begin{array}{l}\text { N.D } \\
\text { N.D } \\
\text { N.D }\end{array}$ \\
\hline 5 & Koko & $\begin{array}{l}1 \\
2 \\
3 \\
\end{array}$ & $\begin{array}{l}7.1 \\
6.9 \\
6.5 \\
\end{array}$ & $\begin{array}{l}0.01 \\
0.04 \\
0.02 \\
\end{array}$ & $\begin{array}{l}\text { N.D } \\
\text { N.D } \\
\text { N.D }\end{array}$ & $\begin{array}{l}\text { N.D } \\
\text { N.D } \\
\text { N.D }\end{array}$ & $\begin{array}{l}\text { N.D } \\
\text { N.D } \\
\text { N.D }\end{array}$ & $\begin{array}{l}\text { N.D } \\
\text { N.D } \\
\text { N.D }\end{array}$ \\
\hline 6 & Oron & $\begin{array}{l}1 \\
2 \\
3\end{array}$ & $\begin{array}{l}5.5 \\
5.2 \\
5.5\end{array}$ & $\begin{array}{l}0.03 \\
0.05 \\
0.02\end{array}$ & $\begin{array}{l}\text { N.D } \\
\text { N.D } \\
\text { N.D }\end{array}$ & $\begin{array}{l}\text { N.D } \\
\text { N.D } \\
\text { N.D }\end{array}$ & $\begin{array}{l}\text { N.D } \\
\text { N.D } \\
\text { N.D }\end{array}$ & $\begin{array}{l}\text { N.D } \\
\text { N.D } \\
\text { N.D }\end{array}$ \\
\hline 7 & Choba & $\begin{array}{l}1 \\
2 \\
3 \\
\end{array}$ & $\begin{array}{l}5.4 \\
5.0 \\
5.3 \\
\end{array}$ & $\begin{array}{l}0.07 \\
0.04 \\
0.05 \\
\end{array}$ & $\begin{array}{l}\text { N.D } \\
\text { N.D } \\
\text { N.D }\end{array}$ & $\begin{array}{l}\text { N.D } \\
\text { N.D } \\
\text { N.D }\end{array}$ & $\begin{array}{l}\text { N.D } \\
\text { N.D } \\
\text { N.D }\end{array}$ & $\begin{array}{l}\text { N.D } \\
\text { N.D } \\
\text { N.D }\end{array}$ \\
\hline 8 & $\begin{array}{c}\text { Mkpana } \\
\text { (Ibeno LGA) }\end{array}$ & $\begin{array}{l}1 \\
2 \\
3\end{array}$ & $\begin{array}{l}6.2 \\
6.0 \\
5.9\end{array}$ & $\begin{array}{l}0.01 \\
0.03 \\
0.05\end{array}$ & $\begin{array}{l}\text { N.D } \\
\text { N.D } \\
\text { N.D }\end{array}$ & $\begin{array}{l}\text { N.D } \\
\text { N.D } \\
\text { N.D }\end{array}$ & $\begin{array}{l}\text { N.D } \\
\text { N.D } \\
\text { N.D }\end{array}$ & $\begin{array}{l}\text { N.D } \\
\text { N.D } \\
\text { N.D }\end{array}$ \\
\hline 9 & $\begin{array}{c}\text { Brass } \\
\text { (Yenegoa) }\end{array}$ & $\begin{array}{l}1 \\
2 \\
3\end{array}$ & $\begin{array}{l}5.8 \\
6.0 \\
6.6\end{array}$ & $\begin{array}{l}0.02 \\
0.04 \\
0.03\end{array}$ & $\begin{array}{l}\text { N.D } \\
\text { N.D } \\
\text { N.D }\end{array}$ & $\begin{array}{l}\text { N.D } \\
\text { N.D } \\
\text { N.D }\end{array}$ & $\begin{array}{l}\text { N.D } \\
\text { N.D } \\
\text { N.D }\end{array}$ & $\begin{array}{l}\text { N.D } \\
\text { N.D } \\
\text { N.D }\end{array}$ \\
\hline
\end{tabular}

\section{Analysis of Residues of Organochlorines in Sediments}

The results of the analyses of organochlorine pesticide residues in sediments from South-South Nigeria are presented in Tables III.

It can be observed in Table III that there was significant difference $(\mathrm{P}<0.05)$ in the levels of the organochlorine pesticides detected in the sediments. The sediment samples from Mkpana Beach in Ibeno Local Government Area of Akwa-Ibom State had the highest frequency of pesticides having lindane $(3.70 \mu \mathrm{g} / \mathrm{kg})$, aldrin $(4.20 \mu \mathrm{g} / \mathrm{kg})$, endosulfan $(0.60 \mu \mathrm{g} / \mathrm{kg})$ and D.D.T $(0.0002 \mu \mathrm{g} / \mathrm{kg})$ residues. It can also be seen that lindane $(1.50 \mu \mathrm{g} / \mathrm{kg})$ as well as aldrin $(30.80$ $\mu \mathrm{g} / \mathrm{kg}$ ) was discovered in sediments from Oron beach in Oron local Government area also in Akwa-Ibom State. The least amount and frequency of pesticide $(1.00 \mu \mathrm{g} / \mathrm{kg})$ of aldrin was only recorded in Agenebode of Etsako-East Local Government Area in Edo State. 
Table-3: Mean Concentration of Organochlorine Pesticide Residues in Sediments of some Water Bodies in South-

\begin{tabular}{|c|c|c|c|c|c|c|c|}
\hline \multicolumn{8}{|c|}{ South } \\
\hline $\mathbf{S} / \mathbf{N}$ & Location & $\begin{array}{l}\text { Sampling } \\
\text { Stations }\end{array}$ & Wt of Extract & $\begin{array}{l}\text { Lindane } \\
(\mu \mathrm{g} / \mathrm{kg})\end{array}$ & $\begin{array}{l}\text { Aldrin } \\
(\mu g / \mathbf{k g})\end{array}$ & $\begin{array}{l}\text { Endosulfan } \\
(\mu \mathrm{g} / \mathrm{kg})\end{array}$ & $\begin{array}{l}\text { DDT } \\
(\mu \mathrm{g} / \mathrm{kg})\end{array}$ \\
\hline 1 & Ekehuan & $\begin{array}{l}1 \\
2 \\
3\end{array}$ & $\begin{array}{l}0.03 \\
0.02 \\
0.03\end{array}$ & $\begin{array}{l}\text { N.D } \\
\text { N.D } \\
\text { N.D }\end{array}$ & $\begin{array}{l}\text { N.D } \\
\text { N.D } \\
\text { N.D }\end{array}$ & $\begin{array}{l}\text { N.D } \\
\text { N.D } \\
\text { N.D }\end{array}$ & $\begin{array}{l}\text { N.D } \\
\text { N.D } \\
\text { N.D }\end{array}$ \\
\hline 2 & Illushin & $\begin{array}{l}1 \\
2 \\
3\end{array}$ & $\begin{array}{l}0.03 \\
0.04 \\
0.02\end{array}$ & $\begin{array}{l}\text { N.D } \\
\text { N.D } \\
\text { N.D }\end{array}$ & $\begin{array}{l}0.10 \pm 0.02^{\mathrm{d}} \\
\text { N.D } \\
\text { N.D }\end{array}$ & $\begin{array}{l}\text { N.D } \\
\text { N.D } \\
\text { N.D }\end{array}$ & $\begin{array}{l}\text { N.D } \\
\text { N.D } \\
\text { N.D }\end{array}$ \\
\hline 3 & Agenebode & $\begin{array}{l}1 \\
2 \\
3 \\
\end{array}$ & $\begin{array}{l}0.06 \\
0.05 \\
0.03\end{array}$ & $\begin{array}{l}\text { N.D } \\
\text { N.D } \\
\text { N.D }\end{array}$ & $\begin{array}{l}1.00 \pm 0.30^{c} \\
\text { N.D } \\
\text { N.D }\end{array}$ & $\begin{array}{l}\text { N.D } \\
\text { N.D } \\
\text { N.D }\end{array}$ & $\begin{array}{l}\text { N.D } \\
\text { N.D } \\
\text { N.D }\end{array}$ \\
\hline 4 & Old NPA & $\begin{array}{l}1 \\
2 \\
3\end{array}$ & $\begin{array}{l}0.07 \\
0.03 \\
0.05\end{array}$ & $\begin{array}{l}\text { N.D } \\
\text { N.D } \\
\text { N.D }\end{array}$ & $\begin{array}{l}\text { N.D } \\
\text { N.D } \\
\text { N.D }\end{array}$ & $\begin{array}{l}\text { N.D } \\
\text { N.D } \\
\text { N.D }\end{array}$ & $\begin{array}{l}\text { N.D } \\
\text { N.D } \\
\text { N.D }\end{array}$ \\
\hline 5 & Koko & $\begin{array}{l}1 \\
2 \\
3 \\
\end{array}$ & $\begin{array}{l}0.07 \\
0.05 \\
0.06\end{array}$ & $\begin{array}{l}\text { N.D } \\
\text { N.D } \\
\text { N.D }\end{array}$ & $\begin{array}{l}\text { N.D } \\
\text { N.D } \\
\text { N.D }\end{array}$ & $\begin{array}{l}\text { N.D } \\
\text { N.D } \\
\text { N.D }\end{array}$ & $\begin{array}{l}\text { N.D } \\
\text { N.D } \\
\text { N.D }\end{array}$ \\
\hline 6 & Oron & $\begin{array}{l}1 \\
2 \\
3\end{array}$ & $\begin{array}{l}0.06 \\
0.04 \\
0.05\end{array}$ & $\begin{array}{l}1.50 \pm 0.25^{\mathrm{a}} \\
\text { N.D } \\
\text { N.D }\end{array}$ & $\begin{array}{l}30.80 \pm 0.20^{\mathrm{a}} \\
\text { N.D } \\
\text { N.D }\end{array}$ & $\begin{array}{l}\text { N.D } \\
\text { N.D } \\
\text { N.D }\end{array}$ & $\begin{array}{l}\text { N.D } \\
\text { N.D } \\
\text { N.D }\end{array}$ \\
\hline 7 & Choba & $\begin{array}{l}1 \\
2 \\
3 \\
\end{array}$ & $\begin{array}{l}0.03 \\
0.06 \\
0.04 \\
\end{array}$ & $\begin{array}{l}\text { N.D } \\
\text { N.D } \\
\text { N.D }\end{array}$ & $\begin{array}{l}\text { N.D } \\
\text { N.D } \\
\text { N.D }\end{array}$ & $\begin{array}{l}\text { N.D } \\
\text { N.D } \\
\text { N.D } \\
\end{array}$ & $\begin{array}{l}\text { N.D } \\
\text { N.D } \\
\text { N.D } \\
\end{array}$ \\
\hline 8 & Mkpana Beach & $\begin{array}{l}1 \\
2 \\
3\end{array}$ & $\begin{array}{l}0.03 \\
0.04 \\
0.07\end{array}$ & $\begin{array}{l}3.70 \pm 0.20^{b} \\
\text { N.D } \\
\text { N.D }\end{array}$ & $\begin{array}{l}4.20 \pm 0.20^{b} \\
\text { N.D } \\
\text { N.D }\end{array}$ & $\begin{array}{l}0.60 \pm 0.30 \mathrm{~d}^{\mathrm{a}} \\
\text { N.D } \\
\text { N.D }\end{array}$ & $\begin{array}{l}0.20 \pm 0.10^{\mathrm{a}} \\
\text { N.D } \\
\text { N.D }\end{array}$ \\
\hline 9 & Brass & $\begin{array}{l}1 \\
2 \\
3\end{array}$ & $\begin{array}{l}0.03 \\
0.03 \\
0.05\end{array}$ & $\begin{array}{l}\text { N.D } \\
\text { N.D } \\
\text { N.D }\end{array}$ & $\begin{array}{l}\text { N.D } \\
\text { N.D } \\
\text { N.D }\end{array}$ & $\begin{array}{l}\text { N.D } \\
\text { N.D } \\
\text { N.D }\end{array}$ & $\begin{array}{l}\text { N.D } \\
\text { N.D } \\
\text { N.D }\end{array}$ \\
\hline
\end{tabular}

*N.D-Not detected

*Means with different superscript were significantly different at $\mathrm{P}<0.05$

\section{DISCUSSION}

\section{Fresh fish}

The results of the analyses of fresh fish samples from water bodies in South-South reveals that no pesticide traces were found in the fresh fish samples from South-South. The results of this study correspond with the report of [13] who analysed Chrysichthys nigrodigitatus, Tilapia guinenses, Mugil cephalus, crayfish (Penaeus sp.), African red snapper (Latjanus agennes) from Lagos lagoon in Nigeria and discovered dichlorodiphenyltrichloroethane, dichlorodiphenyl-dichloroethane (DDD), dichloro-diphenyl-dichloro ethylene (DDE) ranged between 0.023 and 0.069 $\mu \mathrm{g} / \mathrm{g}$. The absence of organochlorine pesticides in fresh fish in this study showed that people in the South-south may have abolished the use of obnoxious fishing methods (pesticides) in fishing maybe as a result of the awareness of threats to the aquatic ecosystem and man.

\section{Water}

This study revealed that water samples from South-south Nigeria had no organochlorine pesticide residues. There were higher amounts of pesticide residues in sediments than fish and water probably because of the hydrophobic tendency of pesticides that makes their existence in water to be in low amounts which makes their evaluation difficult.

The adsorption of organochlorines to sediment is a vital process for their elimination from the water body; ultimately, the sediments will be the final receptacle for organochlorines $[14,15]$.

The results also showed that the people of South-south of Nigeria have probably abolished the use of some obnoxious fishing methods (pesticides) as a result of the awareness of threats to the aquatic ecosystem and man. 


\section{Sediments}

In this study, the amounts of organochlorines in sediments of this study is in line with that of [14] who revealed that sediments are known to serve as a receiving end for the contaminants and could accumulate pesticides overtime.

Since organochlorines have been shown to be willingly absorbed in sediments, they can, serve as a real hazard in the aquatic habitat [16]. The available of pesticides in sediments and not in fish and water could be as a result of the accumulation of the pesticides from the obnoxious fishing methods and wash off of herbicides used in farmlands close to the water bodies that must have settled down over time. The high levels of pesticide residues in sedimentsin Oron, Mkpana, Illushin and Agenebode water bodies above the allowable limit of $0.05 \mu \mathrm{g} / \mathrm{kg}$ recommended by the [17, 18] may be as a result of overtime bioaccumulation of extensive and repeated use of the pesticides in fishing. The presence of these organochlorine pesticides also agrees with the report $[11,19]$.

\section{CONCLUSION}

The outcome of this study have provided insights into the organochlorine pesticides contamination levels in fish, water and sediment samples from some water bodies in South-South, Nigeria. Organochlorine pesticide residues were not found in all the fish and water samples but found in some sediment samples analysed. This depicts the hydrophobic nature of pesticides which means that sediments act as a receiving end for contaminants and hence, have the propensity to accumulate pesticides. The study also reveals that people in this region are gradually abolishing the use of pesticides directly in killing fishes in their water bodies because they may be mindful of the hazards of the use of pesticides in fishing to man and the aquatic ecosystem. The high levels of pesticide residues in sedimentsin Oron, Mkpana, Illushin and Agenebode water bodies above the allowable limit of $0.05 \mu \mathrm{g} / \mathrm{kg}$ recommended by the EPA and USEPA may be as a result of overtime bioaccumulation of extensive and repeated use of the pesticides in fishing.

\section{ACKNOWLEDGEMENT}

This study was supported and funded by the Tertiary Education Fund (TETFUND/NRF2014 "Use of Pesticides in Food Preservation in Nigeria and its Health Implications").

\section{Conflict of Interest}

There is no conflict of interest in relation to this work.

\section{REFERENCES}

1. IUPAC. (1997). Compendium of Chemical Terminology, $2^{\text {nd }}$ ed. (the "Gold Book "). Compiled from (Glossary for chemists of terms used in toxicology (IUPAC Recommendations 1993) on Blackwell scientific publications, Oxford, 2081.

2. Covaci, A., Gheorgheb, A., Voorspelsa, S., Maervoeta, J., Rdekere, E.S., Blustc, R., Schepensa, P. (2005). Polybrominated-Diphenyl-Ethers, polychlorinated-biphenyls and organochlorine pesticides in sediments cores from the western Scheldt River, Belgium). Analytical Aspects and Depth Profiles. Environment International, 31, $367-$ 375.

3. Zhang, G.L.J., Cheng, H.R., LI, X.D., XU, W.H. and Jones, K.C. (2007). Distribution of organochlorine pesticides in the Northern South China Sea: Implications for land outflow and air-sea exchange. Journal of Environmental Science Technology, 41, 3884-3890.

4. Ize-Iyamu, O.K., Asia I.O., Egwakhide P.A. (2007). Concentrations of Residues from Organochlorine Pesticides in Water and Fish from some Rivers in Edo State, Nigeria. International Journal of Physical Science, 2(9), $237-241$.

5. Tanake, S., Iwata, H., Tatsukwa, R. (1994). Global contamination by persistent organochlorine and their ecotoxicological impact on marine mammals. Science Total Environment, 154, 163-177.

6. Shinggu, D.Y., Maitera, O.N., Barminas, J.T. (2014). Levels of Organochlorine Pesticides Residues in Fish, Water and Sediments Biu dam/reservoir Borno State Nigeria. International research journal of pure and applied chemistry, 5(2), 150-159.

7. Yang, R.J.G., Zhoe, Q., Yaun, C., Shi, J. (2005). Occurrence and distribution of organochlorine pesticides (HCH and DDT) in sediments collected from East China Sea. Enviroment International, 31, 799-80.

8. Calades, E.D., Coelho, R.L.C., Souza, K. R., Siba, S.C. (1999). Organochlorine pesticides in Water, Sediments and Fish of Paranoa Lake of Brasilia. Environment, Contamination and Toxicology, 62, 199-206.

9. Osibanjo O., C., Biney D., Calamari N., Kaba I.L., Mbome H., Naeve, P.B.O., Ochumba P.B.O., Saad, M.A.H. (1994). Chlorinated Hydrocarbon Substances. In: Calamari, D. and H. Naeve (Eds.), Review of Pollution in the African Aquatic Environment, CIFA Technical Paper No. 25. FAO, Rome, 61-62.

10. Abolagba, O.J., Igene, J.O., Oronsaye, J.A.O. (2005). Investigation on the Fishing Practices in Ekewan Area of Ovia North East Local Government Areas of Edo State, Nigeria. Journal of Agriculture, Forestry and Fisheries, 6(2), 3540 . 
11. Shinggu, D.Y., Maitora, O.N., Barming, B.J. (2015). Determination of Organochlorine Pesticides Residues in Fish, Water and Sediments in Lake Gorro, Adamawa State, Nigeria, 8(4), 212-220.

12. Darko, G., Akoko, O., Opong, C. (2008). Persistent organochlorine pesticides residue in fish, sediment and water from Lake Bosomtwi, Ghana. Chemosphere, 72, 21-24.

13. Alani, R., Drouillard, K., Olayinka, K., Alo, B. (2013). Bioaccumulation of Organochlorine residues in Fish and invertebrates of Lagos Lagoon, Nigeria. American Journal of Science and Industrial Research, 4(1), 22-30.

14. Ezemonye, L., Ogbeide, O., \& Tongo, I. (2015). Distribution and Ecological Risk Assessment of Pesticide Residues in Surface Water, Sediment and Fish from Ogbesse River, Edo State, Nigeria. Journal of Environmental Chemistry and Ecotoxicology, 7(2): 20-30.

15. William, B.A. (2013). Levels and Distribution of Chlorinated Pesticide Residues in Water and Sediment of Tarkwa Bay, Lagos Lagoon. Journal of Research Environmental Science and Toxicology, 2(1), 1-8.

16. Sitting, M. (1980). Pesticide Manufacturing and Toxic Materials control. Encyclopedia. Park Ridge, Ng, Noyos Data Corporation, 810 .

17. EPA. (1984). Guidelines Establishing Test Procedure for the Analysis of Pollutants under the clean water act. Final rule and proposed rule.

18. United State Environmental Protection Agency (USEPA). (2004). Guidelines for Water Reuse. Camp Dresser and Mckee inc. EPA/625/R-04/108, US.

19. Lanfranchi, A.L., Menone, M.L., Miglioranza, K.S., Janiot, L.J., Aizpun, J.E., Moreno V.J. (2006). Stripped weakfish (Cynoscion guatucupa): a Bio-monitor of Organochlorine Pesticides in Estuarine and near-coastal zones. Marine Pollution bulletin, 52, 74-80. 